\title{
BMJ Open Hydrocolloid dressing as a prophylactic use for hand-foot skin reaction induced by multitargeted kinase inhibitors: protocol of a phase 3 randomised self- controlled study
}

\author{
Sadamoto Zenda (D) , ${ }^{1,2}$ Asako Ryu, ${ }^{3}$ Atsuo Takashima, ${ }^{4}$ Michiko Arai, ${ }^{3}$ \\ Yusuke Takagi, ${ }^{5}$ Tempei Miyaji, ${ }^{2,6}$ Tomoe Mashiko, ${ }^{2}$ Yoichi Shimizu (1) , ${ }^{3}$ \\ Naoya Yamazaki, ${ }^{7}$ Chigusa Morizane, ${ }^{8}$ Takuhiro Yamaguchi, ${ }^{6,9}$ \\ Takashi Kawaguchi, ${ }^{10}$ Akiko Hanai, ${ }^{2}$ Yosuke Uchitomi, ${ }^{2}$ Fukuko Oshiba ${ }^{3}$
}

To cite: Zenda S, Ryu A, Takashima A, et al. Hydrocolloid dressing as a prophylactic use for hand-foot skin reaction induced by multitargeted kinase inhibitors: protocol of a phase 3 randomised selfcontrolled study. BMJ Open 2020;10:e038276. doi:10.1136/ bmjopen-2020-038276

- Prepublication history for this paper is available online. To view these files, please visit the journal online (http://dx.doi. org/10.1136/bmjopen-2020038276).

Received 10 March 2020 Revised 30 July 2020 Accepted 02 September 2020

Check for updates

(C) Author(s) (or their employer(s)) 2020. Re-use permitted under CC BY-NC. No commercial re-use. See rights and permissions. Published by BMJ.

For numbered affiliations see end of article.

Correspondence to Dr Sadamoto Zenda; szenda@east.ncc.go.jp

\section{ABSTRACT}

Introduction Although topical use of moisturisers is slightly effective for the prevention and avoiding the aggravation of hand-foot syndrome induced by multikinase inhibitors, there is still room for improvement. Hydrocolloid dressing is a type of wound dressing often used for wounds such as decubitus ulcers. The purpose of this study is to verify the usefulness of application of hydrocolloid dressings as prophylaxis against development of hand-foot syndrome induced by multikinase inhibitors by comparing the effects of this dressing and standard supportive care (moisturising care alone) within the same individuals.

Methods This study is a phase 3 randomised, selfcontrolled study to compare prophylactic moisturising care with or without hydrocolloid dressing for hand-foot syndrome induced by multikinase inhibitors. Patients with radically unresectable advanced or recurrent colorectal carcinoma, gastrointestinal stromal tumour and hepatocellular carcinoma who scheduled to receive regorafenib or sorafenib therapy are eligible for enrolment.

Supportive care for hand-foot syndrome will consist of basic moisturising care with or without hydrocolloid dressing. If hand-foot syndrome occurs, a steroid ointment will be applied two times per day at the affected sites. The primary endpoint is an incidence rate of grade 2 or more severe hand-foot syndrome (soles of the feet only) assessed by National Cancer Institute ( $\mathrm{NCl}$ ) Common Terminology Criteria for Adverse Events V.4.0. Grading of hand-foot syndrome will be performed by central review using photographs taken weekly by blinded trained physicians. The ethical approval was obtained from National Cancer Center Hospital. The results of this study will be submitted for publication in international peerreviewed journals and the key findings will be presented at international scientific conference.

Discussion If the positive results are found in this study, it is shown that hydrocolloid dressing is effective not only as a symptom management but also as a prevention in handfoot syndrome induced by multikinase.

\section{Strengths and limitations of this study}

- This is a phase 3 randomised, self-controlled study.

- Grading of hand-foot syndrome will be performed by central review using photographs taken weekly by blinded trained physicians to keep objectivity.

- Nurse-led study in which quality assurance is supported by J-SUPPORT trial group.

- This study is not a double-blinded study.

This is not a large-scale study.

Trial status The enrolment was started in January 2019, and planned to closed in January 2021. As of February 2020, 26 patients enrolled in this study.

Trial registration number UMIN Clinical Trial Registry (UMIN000034853).

Protocol version V.1.4, 9 January 2020.

\section{INTRODUCTION}

\section{Background and rationale}

Multikinase inhibitors are oral moleculartargeted drugs that inhibit multiple kinases, ${ }^{1-5}$ including vascular endothelial growth factor receptor, that are involved in tumour growth and angiogenesis, platelet-derived growth factor receptor and rearranged during transfection kinase. Because all these drugs are molecular-targeted drugs, they are associated with minimal systemic adverse effects, unlike the conventional cytotoxic anticancer drugs. However, localised adverse events, particularly hand-foot syndrome or other skin disorders, are known to occur frequently, because these drugs target certain molecules to exert their actions. ${ }^{67}$

Hand-foot syndrome induced by multikinase inhibitor therapy most frequently 


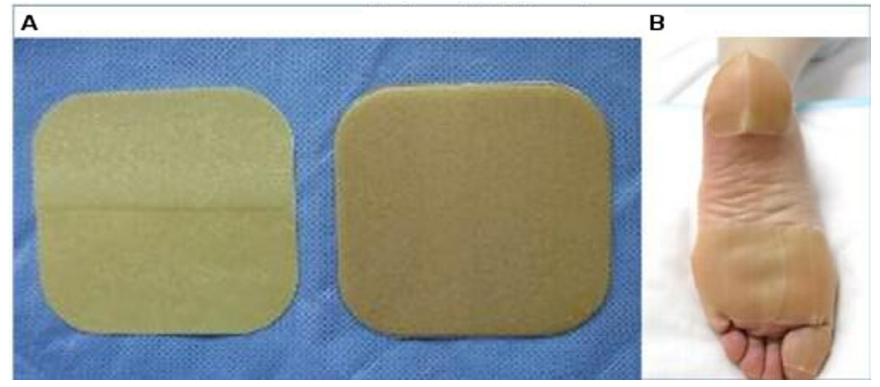

Figure 1 Hydrocolloid dressing. (A) Hydrocolloid dressing. (B) Foot in intervention group was covered by hydrocolloid dressing.

occurred within 1 month of the start of the treatment with regorafenib in the CORRECT Study. ${ }^{6}$

Hand-foot syndrome begins with mild reddening and discomfort, followed by hyperkeratosis, peeling, oedema, blister formation, bleeding and pain (figures 1 and 2). It is not rare for the condition to show serious worsening at the time of the initial response of the underlying disease, and treatment is difficult, with aggravation of the skin disorder often necessitating discontinuation of the anticancer treatment. Because aggravation of hand-foot syndrome is accompanied by pain, the patients' quality of life could markedly deteriorate as a result of the associated difficulty in walking and inability to grip objects. ${ }^{7}$

In regard to evidence for use of topical steroids for the treatment of hand-foot syndrome induced by multikinase inhibitors, their use has been recommended based on a certain supportive study, but the recommendation is based on expert opinion. ${ }^{8}$ There are no reports of prospective clinical studies conducted to determine the usefulness of topical steroids therapy.

A randomised prophylactic administration study of $10 \%$ urea cream and no topical moisturising care in patients with advanced hepatocellular carcinoma receiving

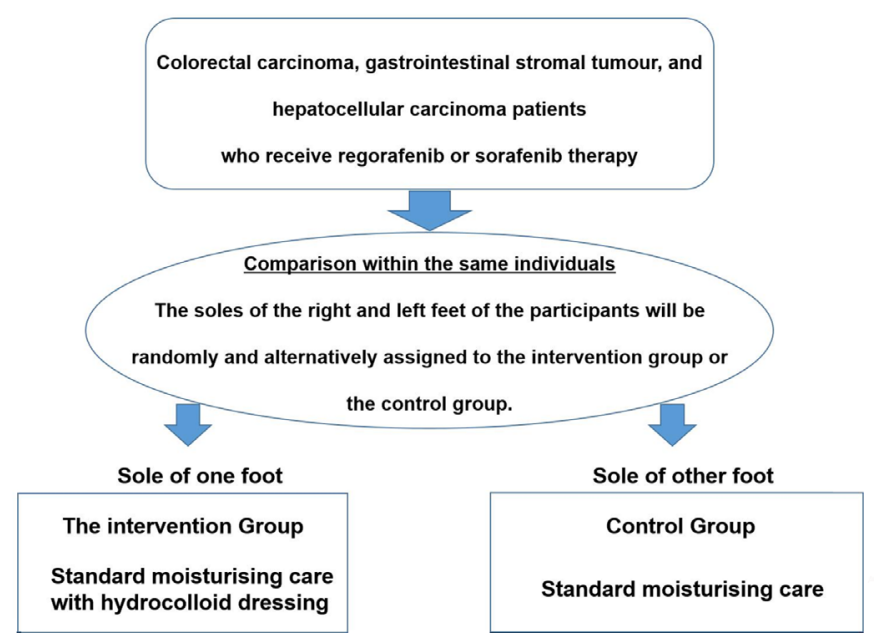

Figure 2 Study schema. Flow chart of the procedures used in this study. This study is a phase 3 randomised, selfcontrolled study to compare prophylactic moisturising care with or without hydrocolloid dressing for hand-foot syndrome induced by multikinase inhibitors. sorafenib therapy reported the occurrence of hand-foot syndrome in $56 \%(\mathrm{n}=439)$ of patients given moisturising care and $73.6 \% \quad(\mathrm{n}=432)$ of those given no moisturising care; thus, the incidence rate was significantly lower $(\mathrm{p}<0.01)$ in the moisturising care group. ${ }^{9}$

Hydrocolloid dressing (figure 1) is a type of wound dressing often used for wounds such as decubitus ulcers. The dressing is composed of a hydrocolloid, which is a polymerised resin with adequate water-absorbing property, and a polyurethane film. It is mainly used for wounds that reach up to the dermis, to protect the wound area and facilitate healing of the wound. In addition, application of the dressing material reduces external friction and shear, potentially alleviating pain.

Shinohara $e t a l^{10}$ reported the results of a randomised multicenter phase 2 trial about hydrocolloid dressing containing ceramide for hand-foot skin reaction on the soles of the feet in metastatic renal cell carcinoma patients treated with sorafenib as a symptom management. In this study, grade 2 or 3 hand-foot syndrome on the soles of the feet was found in $29 \%$ of patients in hydrocolloid dressing arm and was significantly less than the $69 \%$ in control arm $(\mathrm{p}=0.03)$. Hydrocolloid dressing may be effective not only for symptom management but also for the prevention to hand-foot syndrome. Here, we are conducting a phase 3 randomised, self-controlled study to compare prophylactic moisturising care with or without hydrocolloid dressing for hand-foot syndrome induced by multikinase inhibitors

\section{METHODS AND ANALYSIS}

\section{Objective and trial design}

The purpose of this study is to verify the usefulness of application of hydrocolloid dressings as prophylaxis against development of hand-foot syndrome induced by multikinase inhibitors in patients with colorectal carcinoma, gastrointestinal stromal tumour (GIST) or hepatocellular carcinoma receiving treatment with this class of drugs, by comparing the effects of this dressing and standard supportive care (moisturising care alone) within the same individuals (figure 2).

This protocol has been reviewed by the Japan Supportive, Palliative and Psychosocial Oncology Group (J-SUPPORT) and approved as a J-SUPPORT 1701 study.

\section{Patient and public involvement}

Cancer survivors and non-medical persons were included in the members of Scientific Advisory meeting of J-SUPPORT. From protocol concept making, they participated in our discussion.

The enrolment was started in January 2019, and planned to close in January 2021.

\section{Study setting}

This is a single-institution trial (National Cancer Center Hospital, Japan). The institutional review board approved protocol before patient enrolment. 


\section{Eligibility criteria}

Inclusion criteria

Patients fulfilling all of the following criteria and not falling under the exclusion criterion will be eligible for inclusion in this study.

(1) Patients histologically diagnosed as having colorectal carcinoma, GIST or hepatocellular carcinoma (although clinical diagnosis is allowed for hepatocellular carcinoma). There will be no exclusions by the histologic type, (2) patients with radically unresectable advanced or recurrent colorectal carcinoma, GIST or hepatocellular carcinoma, (3) patients who are scheduled to be started on regorafenib or sorafenib therapy, (4) age 20 years or older, (5) perfprmance status (PS) of 0-2 according to the ECOG Scale, (6) patients without cognitive impairment who can communicate verbally, (7) patients with functional gait ability, (8) patients who have no lesions in the soles of the feet (tinea, erythema due to burns or other causes, marked peeling) that could interfere with the evaluation of the skin symptoms and (9) patients willing to provide written informed consent for participation in the study.

\section{Exclusion}

\section{Exclusion criteria}

Patients who have mental disease or psychiatric symptoms interfering with daily life and are judged as being unsuitable for participation in the study.

\section{Intervention}

Intervention as cancer treatment

This study will only include patients scheduled to be treated with regorafenib or sorafenib alone as the study subjects. The appropriate dose regimens, according to guidelines, for the two drugs are as follows.

\section{Regorafenib}

The drug is administered at the dose of $160 \mathrm{mg}$ orally one time a day, after a meal, for 3 consecutive weeks, followed by 1 week of drug withdrawal ( $=1$ course of therapy). The treatment course is repeated. The dose may be reduced, as appropriate, by the doctor in charge according to the patient's condition.

\section{Sorafenib}

This drug is indicated for the treatment of hepatocellular carcinoma.

The drug is administered at the dose of $400 \mathrm{mg}$ orally two times per day for 4 consecutive weeks ( $=1$ course of therapy). The treatment course is repeated. The drug dose may be reduced, as appropriate, by the doctor in charge according to the patient's condition.

\section{Intervention as supportive care arising from cancer treatment} Control arm: basic moisturising care alone

A heparinoid moisturiser will be applied to the soles of the feet two times per day from the start of cancer treatment. If hand-foot syndrome occurs, a steroid ointment (very strong or strongest) will be applied two times per day daily at the affected sites.

Intervention arm: basic moisturising care with hydrocolloid dressing

Adding the basic moisturising care, a hydrocolloid dressing will be applied to the possible sites of development of hand-foot syndrome in the soles of the feet (first toe, base of first toe, base of fifth toe, root of third toe, heel) from the start of cancer treatment.

On the day prior to or on the day of start of the main treatment, after confirming that the soles of the feet are clean, a hydrocolloid dressing will be applied to the predicted site of the hand-foot syndrome in the sole of the foot . Regular application of the hydrocolloid dressing will be continued until all the evaluation procedures in the study have been completed. The duration of continuous application of each dressing will essentially be 1 week. The dressing will be changed and the site will be examined by a nurse on each evaluation day at the outpatient clinic.

If the hydrocolloid dressing becomes detached before the scheduled evaluation day, an extra hydrocolloid dressing given to the patients as reserve should be applied at the site by the patients themselves.

If hand-foot syndrome occurs, a steroid ointment (very strong or strongest) will be applied two times per day daily at the affected sites. A heparinoid moisturising care and the steroid ointment will not be applied at the site of application of the hydrocolloid dressing.

\section{Endpoints}

The primary endpoint is an incidence rate of grade 2 or more severe hand-foot syndrome (soles of the feet only) assessed by National Cancer Institute (NCI) Common Terminology Criteria for Adverse Events (CTCAE) V.4.0. Hand-foot syndrome is defined as palmar-plantar erythrodysesthesia syndrome in CTCAE V.4 and compositely graded by severity of skin changes, pain severity and limitation of activity of daily living. Grading of handfoot syndrome will be performed by central review using photographs taken weekly by blinded trained physicians (figure 3). Pain severity and interference will be accessed by patients using 11-point Numerical Rating Scale and 4-point Likert Scale, respectively.

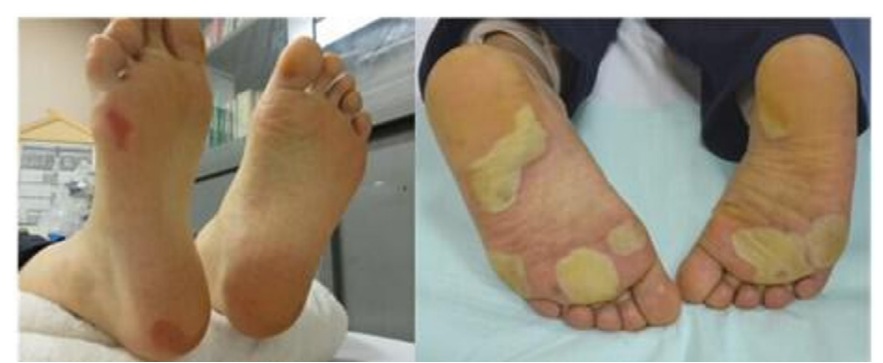

Figure 3 Photo finish for grading of hand-foot syndrome. All the photographs used for a photo finish are taken by the same camera in same light condition room. 


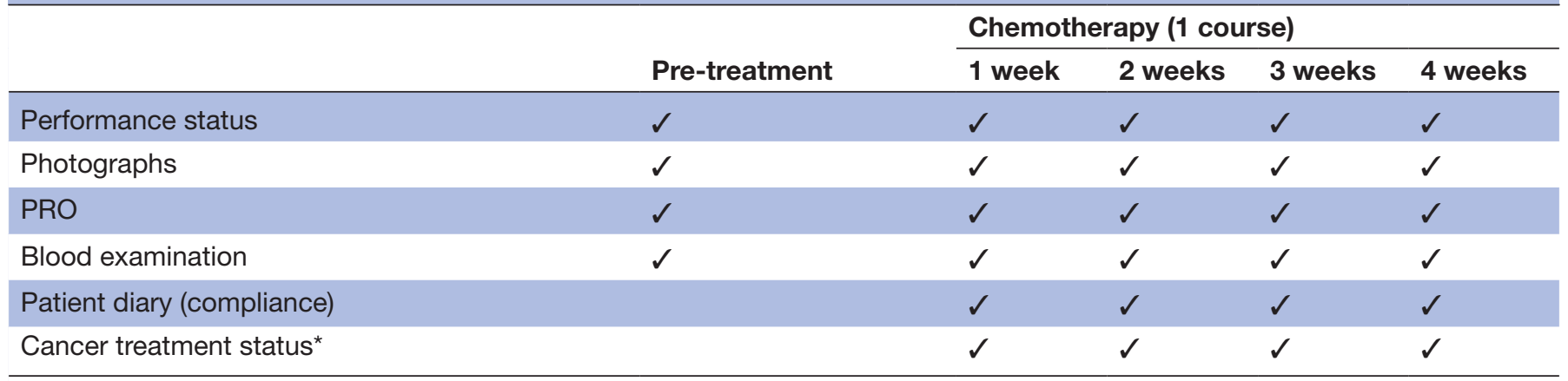

${ }^{*}$ Dose reduction, interruption and/or prolongation.

PRO, patient reported outcome.

Secondary endpoints are the time to first grade 2 or more severe hand-foot syndrome, the incidence rate of grade 1 or more severe hand-foot syndrome, the incidence rate of grade 3 or more severe hand-foot syndrome, the difference in pain score, the frequency of need for modification of regorafenib or sorafenib dose due to the occurrence of hand-foot syndrome and the difference in the score for hand-foot syndrome according to the National Cancer Institute's Patient-Reported Outcomes Version of the CTCAE (NCI PRO-CTCAE). The Japanese version of PRO-CTCAE is linguistically and psychometrically validated. ${ }^{11} 12$

\section{Participant timeline}

After confirming that a patient is to be initiated on oral regorafenib or sorafenib therapy, he/she will be enrolled by the scheduled day of start of the therapy, and the necessary drugs and medical supplies (moisturisers, topical steroids, hydrocolloid dressings) will be prescribed. The soles of the right and left feet will be randomly and alternatively assigned to treatment groups.

Once regorafenib or sorafenib therapy is started, weekly examination is performed, including taking photographs of soles of the feet as well as evaluation of performance status, toxicities other than hand-foot syndrome and process of regorafenib or sorafenib therapy.

The assessment schedule from the initiation of regorafenib or sorafenib therapy to one month after the initiation of that is shown in table 1 .

\section{Sample size}

To justify the cost (about $\$ 8$ per one procedure) of hydrocolloid dressing, we assumed that grade 2 or more HandFoot syndrome (HFS) should be decreased at least $25 \%$ (number needed to treat $=4$ ). We expect the incidence rate of grade 2 or more severe hand-foot syndrome (primary endpoint) to be $5 \%-10 \%$ in the intervention group, and $30 \%-45 \%$ in the control group. The incidence of HFS in the control group was estimated according to the previous study. ${ }^{9}$ The enrolment of 50 patients (100 feet) would provide a power of $80 \%$ to detect $25 \%$ difference between groups with a one-sided significance level of 0.025 in the analysis of the primary endpoint.

\section{Allocation}

After confirming that a patient is to be initiated on oral regorafenib or sorafenib therapy, patients will be enrolled by the scheduled day of start of the therapy, and the necessary drugs and medical supplies (moisturisers, topical steroids, hydrocolloid dressings) will be prescribed.

At enrolment, the soles of the right and left feet will be randomly and alternatively assigned to the test supportive care group and standard supportive care group for intraindividual comparison using minimisation methods. The drugs used for the main treatment (regorafenib, sorafenib) will be set as allocation adjustment factors.

\section{Masking}

This is not a blinded study for clinicians and patients. Trained physicians who evaluate grading of hand-foot syndrome by photographs for central review are blinded.

\section{Data collection methods}

The investigators will maintain individual records for each patient as source data, including a copy of informed consent, medical records, laboratory data, image data, patient diary and other records or note. All data will be collected by the J-SUPPORT Data Center at the National Cancer Center, Center for Public Health Science. Clinical data entry, data management and central monitoring will be performed using electronic data capture (EDC) system, Viedoc 4 (PCG Solutions, Sweden). Patientreported outcomes will be collected using ePRO, Viedoc Me.

\section{Statistical methods of analysis}

Efficacy analysis will include patients who are eligible and enrolled in this study, and must have received at least one dose of regorafenib/sorafenib, one intervention to their feet and one evaluation of HFS. Point estimates and confidence intervals for the incidence of grade 2 or more severe HFS will be calculated and compared between the groups using generalised linear model with robust estimation with adjustment for the allocation factor (anticancer treatments: regorafenib or sorafenib). One-sided significance level will be $0.025 \%$. 


\section{Data management, monitoring and auditing}

Data management and central data monitoring will be performed using the EDC by clinical data manager at the National Cancer Center, Center for Public Health Science. Central data monitoring reports will be compiled by the clinical data managers two times a year and reported to the principal and site investigators. An Independent Data Monitoring Committee has been established which will also review the safety data if serious adverse events occur. An interim analysis will not be performed. Also, auditing is not planned for this study.

\section{Protocol amendments}

Modifications to the study protocol will be communicated to the institutional review board (IRB) at National Cancer Center Hospital. IRB will revise the informed consent materials to be given to participants and adapt them to accord with our institution's guidelines. The protocol was amended as V.1.4 as of January 2020.

\section{Confidentially}

Personal information such as name, address and medical ID will be not collected.

\section{Access to data}

Only clinical data managers at the central data centre have access to reported case data through the EDC system during the study conduct. The data manager will transfer the final data set to the principal investigator and the data will be stored in the electronic format.

\section{Ethics and dissemination}

All patients will be required to provide written informed consent.

This study is performed in accordance with the Ethical Guidelines for Medical and Health Research Involving Human Subjects published by Japan's Ministry of Education, Science, and Technology and the Ministry of Health, Labour, and Welfare and the modified act on the Protection of Personal Information as well as the Declaration of Helsinki. This study was approved by the institutional ethics committee at the National Cancer Center on 30 August 2018 (2018-160). The findings of this trial will be submitted to an international peer-reviewed journal and the key findings will be presented at international scientific conferences. Authorship will be ascribed in accordance with the International Committee of Medical Journal Editors guidance.

\section{DISCUSSION}

In this study, we examined the preventive effect of hydrocolloid dressing on hand-foot syndrome in patients receiving molecular targeted drugs.

This study used randomised within person design, ${ }^{13}$ which was used in the clinical trial to examine the preventative effect of cooling gloves and socks on chemotherapyinduced nail and skin disorders or chemotherapy-induced peripheral neuropathy. ${ }^{14-17}$ Therefore, the primary endpoint is determined by diagnostic image central review and the evaluations are made at a total of four points every week from the start of the treatment to allow the dropouts even the one point affect the patients preference of the treatment. ${ }^{18}$ The effects of attrition bias can be expected to be low because when a participant drops out, both the intervention and the control sides simultaneously decline.

\section{Trial status}

The study is ongoing, and patients are currently being enrolled. Enrolment started in January 2019. As of February 2020, 26 of patients have participated. We thus expect to complete the recruitment by January 2021.

\section{Author affiliations \\ ${ }^{1}$ Radiation Oncology, National Cancer Center Hospital East, Chiba, Japan ${ }^{2}$ Innovation Center for Supportive, Palliative and Psychosocial Care, National Cancer Center Hospital, \& Behavioral and Survivorship Research Group, Center for Public Health Sciences, Tokyo, Japan \\ ${ }^{3}$ Department of Nursing, National Cancer Center Hospital, Chuo-ku, Tokyo, Japan ${ }^{4}$ Department of Gastrointestinal Medical Oncology, National Cancer Center Hospital, Chuo-ku, Tokyo, Japan \\ ${ }^{5}$ Department of Palliative Medicine, Teikyo University School of Medicine Graduate School of Medicine, Itabashi-ku, Tokyo, Japan \\ ${ }^{6}$ Department of Clinical Trial Data Management, Graduate School of Medicine, The University of Tokyo, Bunkyo-ku, Japan \\ ${ }^{7}$ Department of Dermatologic Oncology, National Cancer Center Hospital, Chuo-ku, Tokyo, Japan \\ ${ }^{8}$ Department of Hepatobiliary and Pancreatic Oncology Division, National Cancer Center Hospital, Tokyo, Japan \\ ${ }^{9}$ Division of Biostatistics, Tohoku Graduate School of Medicine, Sendai, Japan ${ }^{10}$ Department of Practical Pharmacy, Tokyo University of Pharmacy and Life Sciences, Tokyo, Japan}

Acknowledgements The authors thank in advance all the patients, investigators and institutions involved in this study. The study protocol was reviewed by J-SUPPRORT Review Committee, supported in part by The National Cancer Center Research and Development Fund (27-A-3,30A-11).

Contributors Study concept was conceived by $S Z$ and $A R$ and design was devised by $\mathrm{YU}, \mathrm{AT}, \mathrm{FO}, \mathrm{AH}$ and TMi. Authors AR and FO were the primary authors of the protocol but all other authors contributed to some part. SZ, AT, CM, YS and NY wrote the main manuscript, and TY, SZ, YU and YT made a manual of double-blinded randomisation and central review system of photographs of hand-foot syndrome. TMi, TK and TMa made an electric data capture system for this trial. All authors read and approved the final manuscript.

Funding This study is supported by Kimura Foundation for Nursing Education (2017) and mainly used in the construction of electric data collection (EDC).

Competing interests None declared.

Patient and public involvement Patients and/or the public were involved in the design, or conduct, or reporting, or dissemination plans of this research. Refer to the Methods section for further details.

Patient consent for publication Not required.

Provenance and peer review Not commissioned; internally peer reviewed.

Open access This is an open access article distributed in accordance with the Creative Commons Attribution Non Commercial (CC BY-NC 4.0) license, which permits others to distribute, remix, adapt, build upon this work non-commercially, and license their derivative works on different terms, provided the original work is properly cited, appropriate credit is given, any changes made indicated, and the use is non-commercial. See: http://creativecommons.org/licenses/by-nc/4.0/.

\section{ORCID iDs}

Sadamoto Zenda http://orcid.org/0000-0002-0421-5805

Yoichi Shimizu http://orcid.org/0000-0002-5154-090X 


\section{REFERENCES}

1 Chu E. An update on the current and emerging targeted agents in metastatic colorectal cancer. Clin Colorectal Cancer 2012;11:1-13.

2 Wilhelm SM, Dumas J, Adnane L, et al. Regorafenib (BAY 73-4506): a new oral multikinase inhibitor of angiogenic, stromal and oncogenic receptor tyrosine kinases with potent preclinical antitumor activity. Int $J$ Cancer 2011;129:245-55.

3 Adnane L, Trail PA, Taylor I, et al. Sorafenib (Bay 43-9006, Nexavar), a dual-action inhibitor that targets Raf/MEK/ERK pathway in tumor cells and tyrosine kinases VEGFR/PDGFR in tumor vasculature. Methods Enzymol 2006;407:597-612.

4 Abrams TJ, Lee LB, Murray LJ, et al. Su11248 inhibits kit and platelet-derived growth factor receptor beta in preclinical models of human small cell lung cancer. Mol Cancer Ther 2003;2:471-8.

5 Mendel DB, Laird AD, Xin X, et al. In vivo antitumor activity of SU11248, a novel tyrosine kinase inhibitor targeting vascular endothelial growth factor and platelet-derived growth factor receptors: determination of a pharmacokinetic/pharmacodynamic relationship. Clin Cancer Res 2003;9:327-37.

6 Grothey A, Van Cutsem E, Sobrero A, et al. Regorafenib monotherapy for previously treated metastatic colorectal cancer (correct): an international, multicentre, randomised, placebocontrolled, phase 3 trial. Lancet 2013;381:303-12.

7 Nardone B, Hensley JR, Kulik L, et al. The effect of hand-foot skin reaction associated with the multikinase inhibitors sorafenib and sunitinib on health-related quality of life. J Drugs Dermatol 2012;11:e61-5.

8 Khan G, Moss RA, Braiteh F, et al. Proactive strategies for regorafenib in metastatic colorectal cancer: implications for optimal patient management. Cancer Manag Res 2014;6:93-103.

9 Ren Z, Zhu K, Kang H, et al. Randomized controlled trial of the prophylactic effect of urea-based cream on sorafenib-associated hand-foot skin reactions in patients with advanced hepatocellular carcinoma. J Clin Oncol 2015;33:894-900.
10 Shinohara N, Nonomura N, Eto M, et al. A randomized multicenter phase II trial on the efficacy of a hydrocolloid dressing containing ceramide with a low-friction external surface for hand-foot skin reaction caused by sorafenib in patients with renal cell carcinoma. Ann Oncol 2014;25:472-6.

11 Miyaji T, lioka Y, Kuroda Y, et al. Japanese translation and linguistic validation of the US National cancer Institute's patient-reported outcomes version of the common terminology criteria for adverse events (PRO-CTCAE). J Patient Rep Outcomes 2017;1:8.

12 Kawaguchi T, Azuma K, Sano M, et al. The Japanese version of the National cancer Institute's patient-reported outcomes version of the common terminology criteria for adverse events (PRO-CTCAE): psychometric validation and discordance between clinician and patient assessments of adverse events. J Patient Rep Outcomes 2017;2:2.

13 Pandis N, Chung B, Scherer RW, et al. Consort 2010 statement: extension checklist for reporting within person randomised trials. $\mathrm{Br}$ J Dermatol 2019;180:534-52.

14 Scotté F, Tourani J-M, Banu E, et al. Multicenter study of a frozen glove to prevent docetaxel-induced onycholysis and cutaneous toxicity of the hand. J Clin Oncol 2005;23:4424-9.

15 Scotté F, Banu E, Medioni J, et al. Matched case-control phase 2 study to evaluate the use of a frozen sock to prevent docetaxelinduced onycholysis and cutaneous toxicity of the foot. Cancer 2008;112:1625-31.

16 Ishiguro H, Takashima S, Yoshimura K, et al. Degree of freezing does not affect efficacy of frozen gloves for prevention of docetaxelinduced nail toxicity in breast cancer patients. Support Care Cancer 2012;20:2017-24.

17 Hanai A, Ishiguro H, Sozu T, et al. Effects of cryotherapy on objective and subjective symptoms of paclitaxel-induced neuropathy: prospective self-controlled trial. J Natl Cancer Inst 2018;110:141-8.

18 Gewandter JS, Brell J, Cavaletti G, et al. Trial designs for chemotherapy-induced peripheral neuropathy prevention: ACTTION recommendations. Neurology 2018;91:403-13. 